\title{
Heterologous prime-boost regimens with HAdV-5 and NDV vectors elicit stronger immune responses to Ebola virus than homologous regimens in mice
}

\author{
Wei Zhao ${ }^{1}$ Peng Zhang ${ }^{1} \cdot$ Shuang Bai ${ }^{1} \cdot$ Min $\mathrm{Lv}^{1} \cdot$ Jian Wang ${ }^{1} \cdot$ Weixin Chen ${ }^{1} \cdot$ Qingzhong $\mathrm{Yu}^{2} \cdot$ Jiang Wu$^{1}$ (1)
}

Received: 10 May 2021 / Accepted: 23 July 2021 / Published online: 30 September 2021

( ) The Author(s), under exclusive licence to Springer-Verlag GmbH Austria, part of Springer Nature 2021

\begin{abstract}
The 2014 Ebola outbreak in West Africa resulted in more than 11,000 deaths, highlighting the need for a vaccine. A phase I clinical trial of a human adenovirus type 5 vector-based Ebola virus (EBOV) vaccine (HAdV-5-MakGP) showed that a homologous prime-boost regimen with HAdV-5 vaccine elicited a robust humoral response but a weak cellular immune response. Due to pre-existing anti-vector immunity, boosting with the same vaccine did not increase the magnitude of the cellular immune response, which contributes significantly to protection against EBOV infection. Here, we generated a recombinant Newcastle disease virus (NDV), based on the LaSota vaccine strain, expressing the GP protein of the EBOV variant Makona (rLS/EB-GP) using reverse genetics technology. The humoral and cellular immune responses to this vaccine candidate in mice immunized using a homologous prime-boost regimen or a heterologous prime-boost regimen with the HAdV-5-vectored Ebola vaccine were assessed using ELISA and ELISPOT assays. The ELISA and ELISPOT results showed that mice primed with rLS/EB-GP and boosted with HAdV-5-MakGP (NDV+HAdV-5) or, reversed, primed with HAdV-5-MakGP and boosted with rLS/EB-GP (HAdV-5+NDV) exhibited more-potent EBOV GP-specific antibody and cellular immune responses than those receiving the same vaccine twice. The most robust EBOV GP-specific antibody and T-cell responses were detected in the HAdV-5-MakGP-primed and rLS/EB-GP-boosted (HAdV-5+NDV) mice. These results suggest that the HAdV-5 prime-NDV boost regimen is more effective in stimulating EBOV-specific immunity than homologous regimens alone, indicating the potential boosting ability of the NDV vector in human vaccine use.
\end{abstract}

\section{Introduction}

The 2013-16 Ebola outbreak in West Africa was the most devastating human infectious disease witnessed in recent times, causing over 28,000 reported cases and more than 11,000 deaths [7]. Recent studies have revealed that the Ebola virus (EBOV) spreads by human-to-human

Handling Editor: Tim Skern.

Jiang Wu

wj81732@hotmail.com

1 Institute for Immunization and Prevention, Beijing Center for Disease Prevention and Control, Beijing Research Center for Preventive Medicine, No. 16, Hepingli Middle Street, Dongcheng District, Beijing 100013, China

2 United States Department of Agriculture. Southeast Poultry Research Laboratory, National Poultry Research Center, Agricultural Research Services, 934 College Station Road, Athens, GA 30605, USA transmission through contact with body fluids of infected people; even the recovered can still transmit the virus via their semen for up to 7 weeks after recovery from illness [5]. Due to the ease of transmission and lack of approved antiviral therapy, further small outbreaks could occur at any time. This threat highlights the need for a vaccine to prevent infection and avoid repetition.

In 2017, the China FDA approved an Ebola vaccine based on a human adenovirus serotype $5(\mathrm{HAdV}-5)$ vector. A phase I clinical trial of the HAdV-5-vectored Ebola vaccine in China has shown that two doses of the homologous HAdV-5 Ebola vaccine with a six-month interval elicit a robust humoral response but only a weak cellular immune response [17]. Unlike the induction of antibodies, repeated homologous boosting with the same vaccine does not increase the magnitude of the cellular immune response, as pre-existing anti-vector immunity inhibits antigen processing [13]. The suppression of immune responses caused by HAdV-5 pre-existing immunity (PEI) to the homologous vaccine boost has also been extensively reported in animal 
models and clinical trials $[3,27]$. To address this concern, researchers have attempted to develop a heterologous prime-boost immunization strategy. Several pre-clinical and clinical studies have shown that heterologous prime-boost regimens generate stronger and broader immune responses and provide significant durability benefits when compared to homologous boosting [21, 24, 25]. These advantages of heterologous prime-boost regimens over homologous regimens might be due to their ability to avoid the consequences of pre-existing anti-vector immunity. The heterologous prime-boost strategy delivers the same or similar antigens using different vaccine vectors, which redirects the immune response to the antigens rather than the vectors. Because of the highlighted potential mentioned above, such a strategy represents a new way of immunization and has been applied to the development of vaccines against a wide range of pathogens [13].

Newcastle disease virus (NDV) is a non-segmented, single-stranded negative-sense RNA virus belonging to the genus Orthoavulavirus, family Paramyxoviridae [23]. Several characteristics make NDV a promising vaccine vector against human and veterinary pathogens. NDV is safe for administration to humans, due to a natural host range restriction [8]. In clinical trials for cancer treatment, NDV as an oncolytic agent was well tolerated without causing adverse effects [11]. Since NDV is an avian virus that is antigenically distinct from human pathogens, there is no preexisting immunity in humans that would limit the utility of an NDV vaccine vector [1]. Furthermore, NDV replicates in the respiratory tract and induces strong humoral and cellular immune responses, both at the mucosal and systemic levels, in murine and nonhuman primate models, thus making this vector an attractive candidate for vaccine development $[1$, 15].

In the present study, we generated an infectious clone of the NDV LaSota strain as a vaccine vector, inserted the surface glycoprotein (GP) gene of EBOV into the infectious clone, and rescued a recombinant NDV that expresses the GP protein of the EBOV variant Makona. Evaluation of the NDV-vectored Ebola vaccine in mice through different immunization regimens with the HAdV-5-vectored Ebola vaccine allowed us to better understand the immune responses elicited after heterologous and homologous prime-boost regimens.

\section{Materials and methods}

\section{Cell lines, virus, and RNA preparation}

HEp-2, DF-1, and Vero cell lines, obtained from the American Type Culture Collection (Manassas, VA), were maintained in Dulbecco's minimal essential medium (DMEM) supplemented with $10 \%$ fetal bovine serum (FBS). The modified vaccinia virus vTF7-3, expressing the T7 RNA polymerase, was obtained from ATCC (VR-2153; ATCC) and was grown in primary chicken embryo fibroblast cells. The NDV LaSota strain obtained from the China Veterinary Culture Collection Center was propagated in 9-day-old embryonated specific-pathogen-free (SPF) chicken eggs as a stock. Total RNA was extracted from the allantoic fluid of the infected eggs at $72 \mathrm{~h}$ post-inoculation using TRIzol Reagent (Invitrogen).

\section{Construction of a full-length cDNA clone of the NDV LaSota strain}

The construction of a plasmid carrying the full-length antigenomic cDNA of the NDV LaSota strain has been described previously [31]. In brief, RNA was extracted and subjected to RT-PCR with four pairs of specific primers using a Superscript ${ }^{\mathrm{TM}}$ III One Step RT-PCR System with Platinum Taq High Fidelity (Invitrogen) to generate four overlapping PCR fragments of the entire viral genome (Fig. 1). These four cDNA fragments were sequentially cloned into a modified pBluescript vector using an InFusion ${ }^{\circledR}$ PCR Cloning Kit (Clontech) to construct a complete genomic cDNA of the LaSota virus. The final plasmid containing the full-length LaSota strain genome, designated as pFLC-LS, was amplified in Stbl 2 cells at $30^{\circ} \mathrm{C}$ and purified using a QIAprep Spin Miniprep Kit (QIAGEN, Valencia, CA).

\section{Construction of NP, $P$, and $L$ gene expression plasmids}

Viral rescue from infectious clones for members of the paramyxovirus family requires the co-expression of the viral nucleoprotein $(\mathrm{NP})$, phosphoprotein $(\mathrm{P})$, and polymerase (L) genes [9]. Amplicons containing the open reading frames (ORFs) of the NP, P, and L genes were generated by RT-PCR from the virus genome and cloned into a modified pBluescript vector using an In-Fusion PCR Cloning Kit (Clontech). The resulting plasmid constructs were designated as $\mathrm{p}-\mathrm{NP}$, $\mathrm{p}-\mathrm{P}$, and $\mathrm{p}-\mathrm{L}$, respectively.

\section{Construction of a recombinant CDNA clone containing the GP gene of EBOV}

The plasmid containing the GP gene of the EBOV Makona strain was a kind gift from Dr. Xu, Beijing Institute of Biotechnology [28]. The procedure for construction of the recombinant cDNA clone containing the GP gene of EBOV is illustrated in Fig. 1. Briefly, a cDNA fragment representing the ORF encoding the EBOV GP was amplified using paired specific primers into which gene end-intergenic-gene 


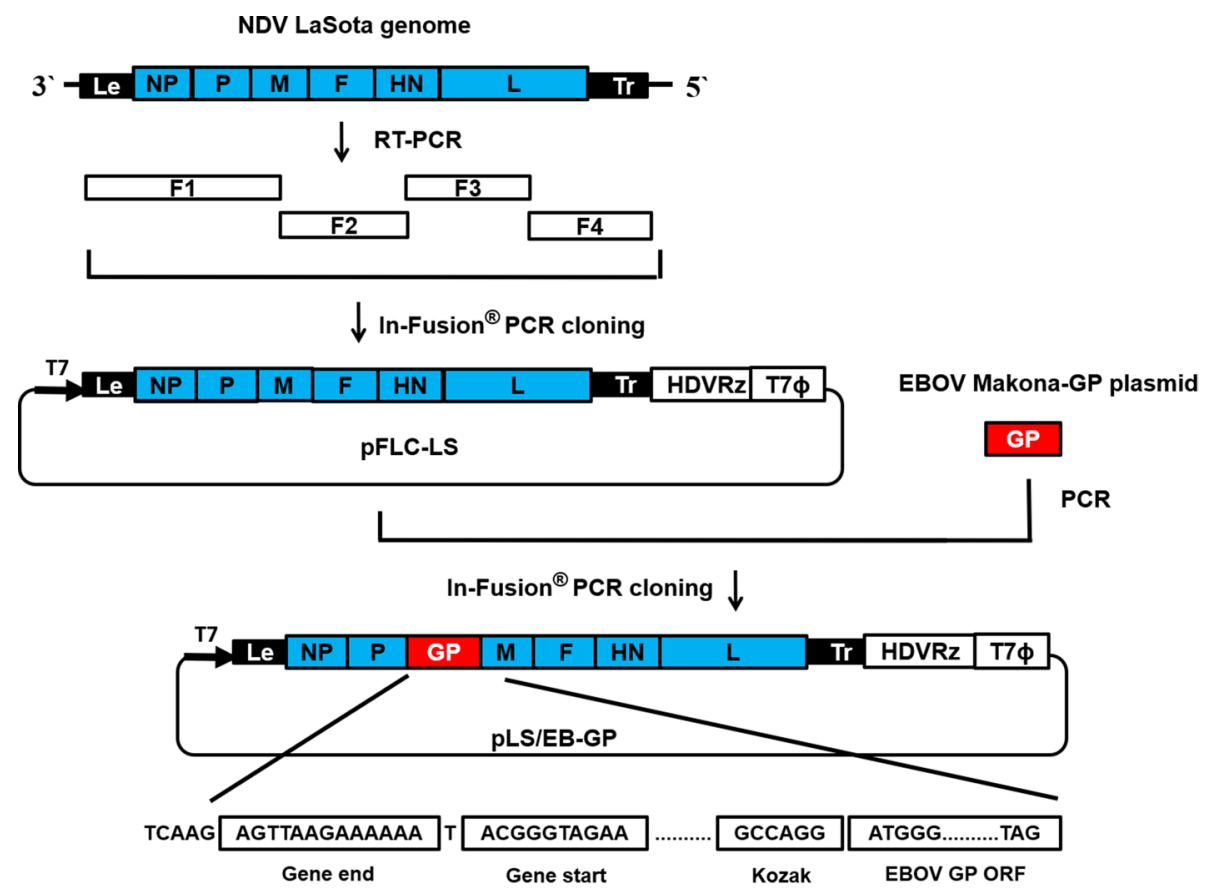

Fig. 1 Schematic representation of the recombinant cDNA clone containing the GP gene of EBOV. Four cDNA fragments covering the complete sequence of the LaSota strain genomic RNA were generated and cloned into a pBlueScript-based modified plasmid. The open reading frame (ORF) of the EBOV GP gene along with the NDV trans-acting signal sequences was amplified and inserted into

start motifs sequences were incorporated. An optimal Kozak translation sequence was inserted upstream of the GP gene translation start codon to enhance foreign gene expression. Subsequently, the PCR product containing the GP ORF along with the NDV trans-acting signal sequences was introduced into the P-M intergenic region of the pFLC-LS vector using an In-Fusion ${ }^{\circledR}$ PCR Cloning Kit (Clontech). The resulting recombinant cDNA clone was designated as pLS/EB-GP.

\section{Virus rescue and propagation}

The procedures for transfection of HEp-2 cells with the full-length cDNA clone, pFLC-LS or pLS/EB-GP, and the supporting plasmids, $\mathrm{p}-\mathrm{NP}, \mathrm{p}-\mathrm{P}$, and $\mathrm{p}-\mathrm{L}$, were described previously [32]. HEp-2 cells grown in a six-well plate were infected with vTF7-3 at a multiplicity of infection (MOI) of 3. Subsequently, the cells were cotransfected with $2 \mu \mathrm{g}$ of pFLC-LS or pLS/EB-GP together with $1 \mu \mathrm{g}$ of $\mathrm{p}-\mathrm{NP}, 0.5 \mu \mathrm{g}$ of p-P, and $0.1 \mu \mathrm{g}$ of p-L using $6 \mu \mathrm{l}$ of Lipofectamine ${ }^{\mathrm{TM}} 2000$ (Invitrogen). After $6 \mathrm{~h}$ of incubation at $37^{\circ} \mathrm{C}$, the medium was replaced with DMEM containing $2 \% \mathrm{FBS}$, and the cells were incubated for three days at $37^{\circ} \mathrm{C}$. The supernatants were then inoculated into the allantoic cavities of 10-dayold embryonated SPF eggs. After 4 days of incubation, the the pFLC-LS vector in the non-coding region downstream of the $\mathrm{P}$ gene, resulting in the $\mathrm{pLS} / \mathrm{EB}-\mathrm{GP}$ clone. The direction of the T7 promoter is indicated by a bold black arrow. HDVRz and T7 $\Phi$ represent the site of the hepatitis delta virus ribozyme and the T7 terminator sequence, respectively. The NDV gene start and gene end sequences, Kozak sequence, and the EBOV GP ORF are boxed

allantoic fluid was harvested, and the rescued viruses (rLS and rLS/EB-GP) were screened by hemagglutination assay (HA). The nucleotide sequences of the rescued viruses were determined by sequencing the RT-PCR products amplified from the viral genomes as described previously [12].

\section{Immunofluorescence assay (IFA)}

The procedure for IFA was described previously [31]. Vero cells in 6-well plates were infected with rLS or rLS/EB-GP at an MOI of 0.01 . The primary antibody was rabbit antiEBOV GP protein antibody (Sino Biological Inc, Beijing, China). The secondary antibody was fluorescein (FITC)conjugated goat anti-rabbit $\operatorname{IgG}(\mathrm{H}+\mathrm{L})$ (Thermo Fisher). Fluorescence images were observed under an inverted fluorescence microscope at $100 \times$ magnification with a matching wavelength filter for FITC.

\section{Prime-boost regimens and animal experiments}

The replication-defective HAdV-5-MakGP vaccine was developed and prepared by the Beijing Institute of Biotechnology and Tianjin CanSino Biotechnology as described previously [33]. 
A group of 4- to 6-week old female BALB/c mice was obtained from Beijing Vital River Laboratory Animal Co. Ltd (Beijing, China) and housed in an animal biosafety level 2 facility. The mice were confirmed by ELISA to be free of EBOV antibodies prior to immunization and then randomly divided into six groups of six mice each and inoculated with the initial dose of vaccine on day 0 and the booster on day 21, as illustrated in Fig. 2. Mice in the HAdV-5 group were vaccinated intramuscularly (i.m.) with $1 \times 10^{7}$ infectious units (IFU) of HAdV-5-MakGP on days 0 and 21. Mice in the NDV group were inoculated intranasally (i.n.) with 1 $\times 10^{6}$ plaque-forming units (PFU) of rLS/EB-GP on days 0 and 21 . Mice in the HAdV-5+NDV group were primed by i.m. injection with $1 \times 10^{7}$ IFU of HAdV-5-MakGP on day 0 , followed by i.n. boosting with $1 \times 10^{6} \mathrm{PFU}$ of rLS/ EB-GP on day 21. Conversely, mice in the NDV+ HAdV-5 group were primed i.n. with $1 \times 10^{6} \mathrm{PFU}$ of rLS/EB-GP and then boosted by i.m. injection with $1 \times 10^{7} \mathrm{IFU}$ of HAdV5 -MakGP. Mice in the rLS group were vaccinated i.n. with $1 \times 10^{6}$ PFU of rLS and served as LaSota vector controls. Mice in the PBS group were inoculated with $100 \mu 1$ of PBS via the intranasal route and served as unvaccinated controls.

At 21 and 49 days post-immunization (PI), blood was collected from each mouse, and serum was obtained by centrifugation of whole blood. Isolated sera were transferred to labeled tubes and stored at $-20^{\circ} \mathrm{C}$ until analysis. Eighteen mice from each group were humanely euthanized at day 49 , and splenocytes were isolated for ELISPOT assay.

\section{EBOV GP ELISA}

96-well polystyrene microtiter plates (Costar, Bethesda, $\mathrm{MD})$ were coated overnight at $2-8^{\circ} \mathrm{C}$ with $2 \mu \mathrm{g}$ of EBOV GP protein (subtype Zaire, strain H. sapiens-wt/GIN/2014/ Kissidougou-C15) (Sino Biological Inc, Beijing, China) per ml. Plates were washed with PBS containing $0.05 \%$ Tween

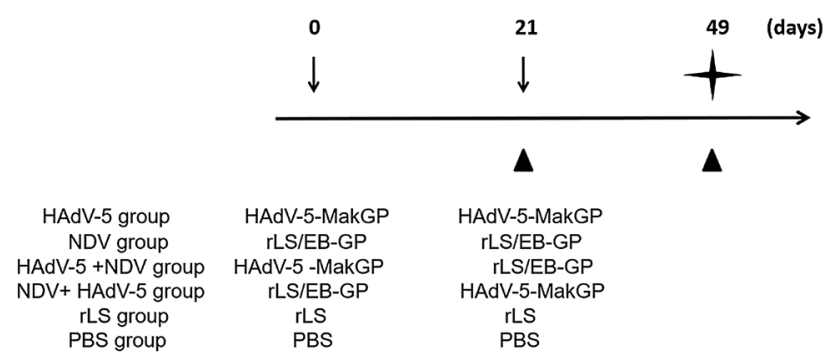

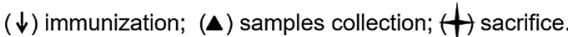

Fig. 2 Schemes for animal immunization and sample collection. $\mathrm{BALB} / \mathrm{c}$ mice were divided randomly into six groups and were immunized at day 0 (prime) and day 21 (boost). Serum samples were collected at day 21 and 49 PI for ELISA assay. Mice were sacrificed at day $49 \mathrm{PI}$, and splenocytes were isolated for ELISPOT assay
(PBST) and blocked with 5\% goat serum for $1 \mathrm{~h}$ at $37^{\circ} \mathrm{C}$. After blocking, twofold serial dilutions of sera were added to EBOV-GP-protein-coated plates in duplicate and incubated for $1 \mathrm{~h}$ at $37^{\circ} \mathrm{C}$. The bound antibodies were detected using horseradish-peroxidase-conjugated goat anti-Mouse IgG antibody (Thermo Fisher). After a 1-h incubation, color was developed by adding TMB (Solarbio, Beijing, China) peroxidase substrate, and absorbance was read at $450 \mathrm{~nm}$ in a Bio-Rad 550 ELISA plate reader (Bio-Rad, Hercules, CA). The antibody titer was expressed as the reciprocal of the highest antibody dilution that yielded greater than or equal to 2.1 times the background absorbance of PBS (the negative control) [19].

\section{ELISPOT assay}

A commercial kit (mouse IFN- $\gamma$ precoated ELISPOT kit, Dakewe Biotech, Shenzhen, China) was used for the ELISPOT assay. Lymphocytes from the spleen were harvested from each mouse, and a single cell suspension $\left(5 \times 10^{5}\right.$ cells/well) was prepared and stimulated with $20 \mu \mathrm{g}$ of the EBOV GP protein described above per ml. After incubation at $37^{\circ} \mathrm{C}$ for $24 \mathrm{~h}$, the cells were removed and the plates were processed according to the manufacturer's instructions. The number of spots was determined automatically using an automatic CTL Immunospot ${ }^{\circledR}$ Reader (Cellular Technology, Shaker Heights, Ohio).

\section{Statistical analysis}

Statistical analysis was performed using GraphPad Prism. All data were shown as the mean and standard deviation. Data were statistically analyzed using the unpaired, twotailed Student's $t$-test. $p$-values of $<0.05$ were considered significant.

\section{Results}

\section{Generation of the rLS and rLS/EB-GP viruses}

A full-length cDNA clone encoding the complete antisense genome of the NDV LaSota strain was constructed by assembling four overlapping PCR fragments through PCR and In-Fusion PCR cloning, as illustrated in Fig. 1. The GP gene of EBOV flanked by the NDV trans-acting elements was inserted into the P-M intergenic region of the LaSota full-length clone as an additional transcription unit, which increased the length of the recombinant clone by 2232 nucleotides (nt). Thus, the total length of the cDNA clone in the pLS/EB-GP plasmid was 17,418 nt and therefore divisible by 6 , conforming to the "Rule of Six" [16]. Then, the NDV LaSota virus and the recombinant virus, designated as rLS 
and rLS/EB-GP, respectively, were rescued from the cDNAs as described in Materials and methods. Sequencing analysis confirmed the nucleotide sequence fidelity of the rescued viruses (Fig. 3).

\section{Expression of the EBOV GP protein by rLS/EB-GP}

To examine the expression of EBOV GP protein by rLS/ EB-GP, Vero cells were infected with rLS/EB-GP at an MOI of 0.01 . After $48 \mathrm{~h}$, the cells were fixed and incubated with a rabbit antiserum directed against the EBOV GP protein, followed by immunostaining with FITC-conjugated goat anti-rabbit IgG. Positive staining was observed in rLS/EBGP-infected Vero cells using an anti-EBOV GP antibody and an FITC-labeled secondary antibody (Fig. 4a). In contrast, no fluorescence was detected in NDV-LaSota-infected cells after staining with the same antiserum and FITC conjugate (Fig. 4b). This result demonstrated that the EBOV GP protein was expressed from the rLS/EB-GP-recombinantinfected cells.

\section{Heterologous prime-boost regimens elicit more-robust EBOV-GP-specific antibody responses than homologous regimens}

To assess the effectiveness of heterologous prime-boost vaccination regimens with rLS/EB-GP and HAdV-5-MakGP for inducing immune responses, BALB/c mice were immunized on day 0 (prime) and day 21 (boost) with these vectored EBOV vaccines as illustrated in Fig. 2. EBOV-GP-specific antibodies in the collected sera were detected by ELISA. As shown in Fig. 5, although EBOV-GP-specific IgG titers were relatively low in all groups after the first immunization (prime), they increased dramatically after the second immunization (boost). The heterologous prime-boost with HAdV-5 and NDV induced a significantly higher geometric mean titer (GMT) of GP-specific IgG at day 49 post-immunization (PI) than the homologous prime-boost regimens (HAdV-5+NDV group vs. HAdV-5 group, $p<0.05$; HAdV$5+\mathrm{NDV}$ group vs. NDV group, $p<0.05$ ). The mice in the $\mathrm{NDV}+\mathrm{HAdV}-5$ group produced approximately 1.5 -fold and 3.1-fold higher GP-specific IgG titers than those receiving the NDV and HAdV-5 homologous regimens, respectively. These results demonstrated that the heterologous primeboost regimens could elicit more-robust EBOV-GP-specific antibody responses than the homologous regimens.

\section{Heterologous prime-boost regimens elicit stronger cellular immune responses than homologous regimens}

Splenocytes isolated from the immunized mice at day 49 PI were assayed by IFN- $\boldsymbol{\gamma}$-specific ELISPOT to analyze the antigen-specified T-cell responses induced by the different

A

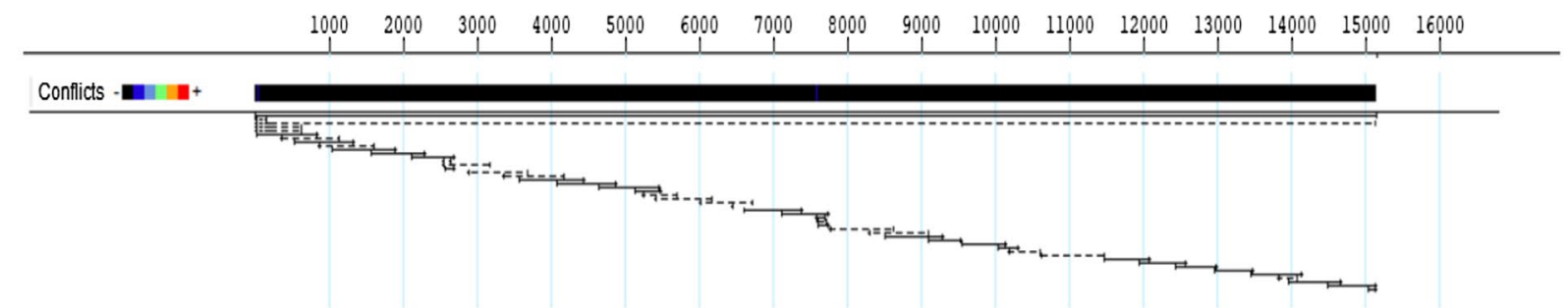

B

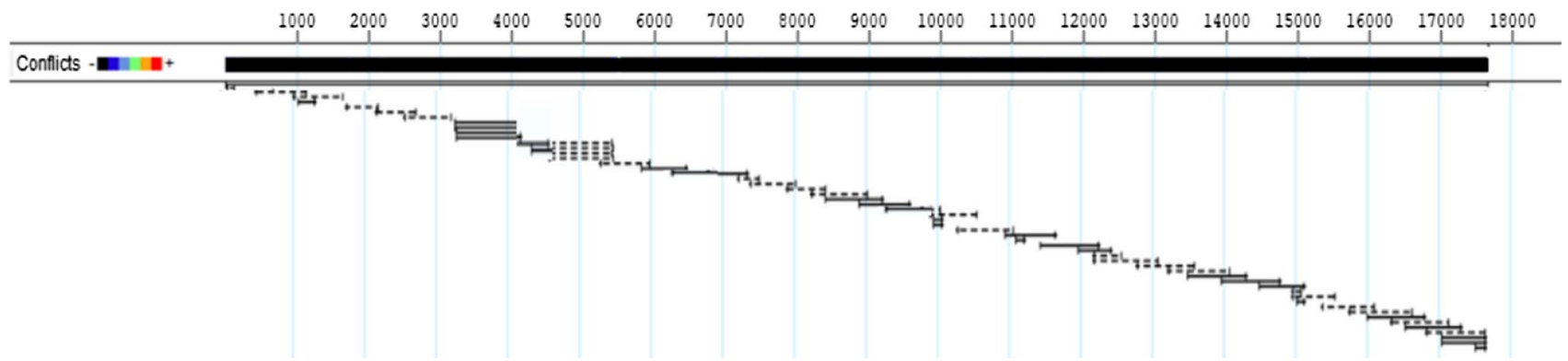

Fig. 3 Sequencing analysis of the rescued viruses. The RT-PCR products generated from the rescued viruses rLS (A) and rLS/EB-GP (B) were sequenced directly with 32 and 37 pairs of gene-specific prim- ers, respectively. Nucleotide sequence editing, assembly, and comparison analysis were carried out using the DNASTAR Lasergene software (DNASTAR, Madison, WI, USA) 
Fig. 4 Detection of EBOV GP protein expression by IFA. Vero cells were infected with rLS or rLS/EB-GP at an MOI of 0.001 . At 48 hours postinfection, the cells were fixed and stained with a rabbit anti-EBOV GP protein antibody, followed by an FITC-labeled goat antirabbit IgG secondary antibody. Cytopathic effect (CPE) and fluorescence images in the same field of the infected cells were photographed using an inverted fluorescence microscope at $100 \times$ magnification under visible light for CPE or a UV light with a matching wavelength filter for FITC
CPE

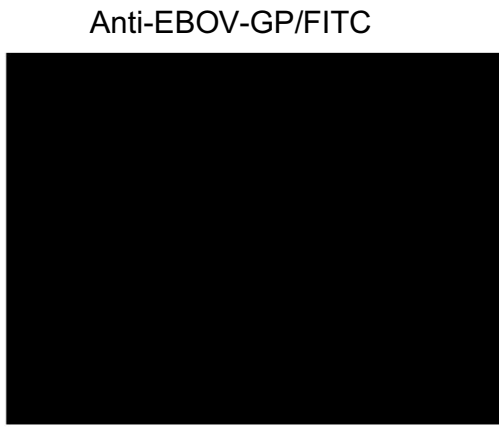

rLS
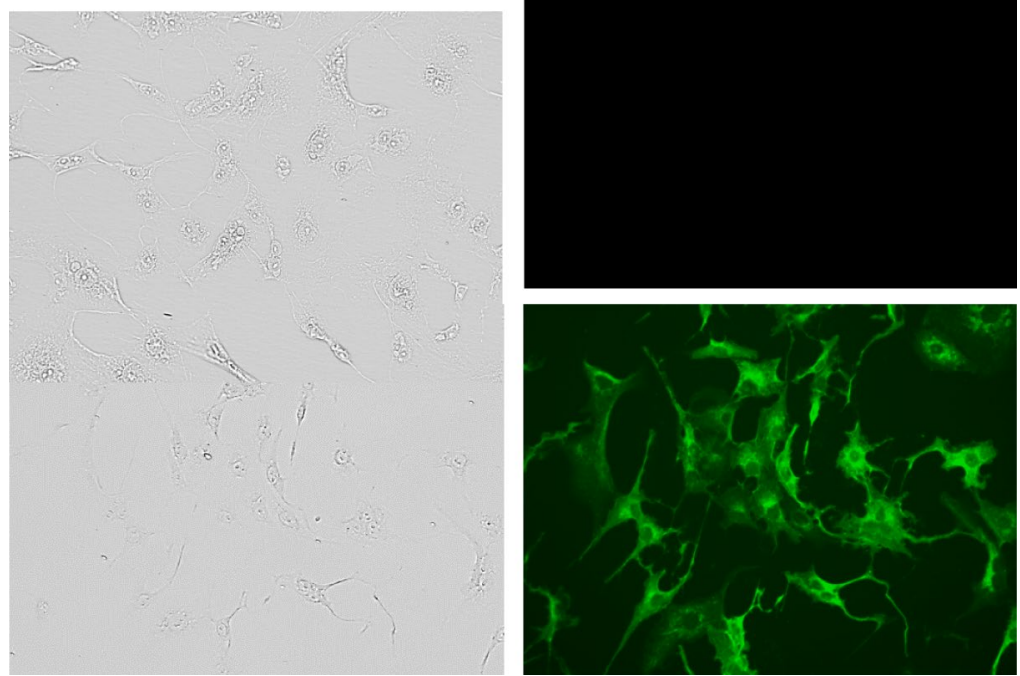

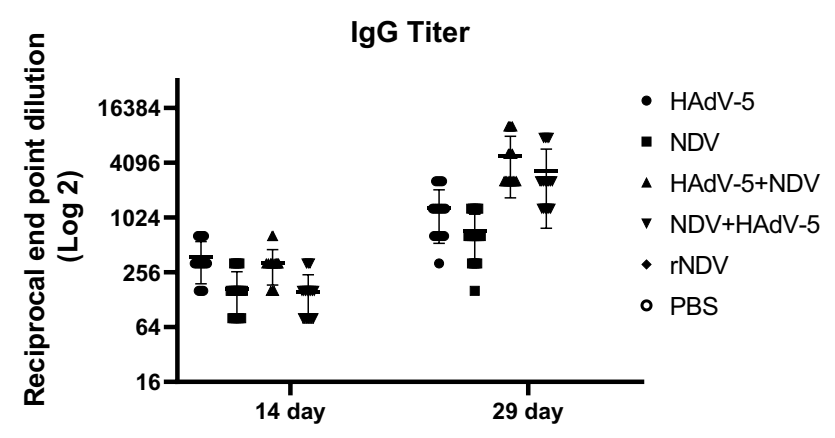

Fig. 5 EBOV-GP-specific antibody responses. Serum samples were collected from each mouse at 21 and 49 days post-inoculation. Serum EBOV-GP-specific IgG antibodies were determined by ELISA and used to calculate the GMT for each group of mice

immunization regimens. Interestingly, as shown in Fig. 6, the magnitude of the IFN- $\gamma$-specific ELISPOT responses correlated positively with the antibody responses in all immunization groups. Specifically, splenocytes obtained from mice in the heterologous HAdV-5+NDV and $\mathrm{NDV}+\mathrm{HAdV}-5$ regimen groups exhibited significantly higher IFN- $\gamma$ expression than those from mice in the homologous HAdV-5 regimen $(p<0.05)$ and rLS/EB-GP regimen $(p<0.05)$ groups. The IFN- $\gamma$-specific ELISPOT data, together with the antibody response results, demonstrate that mice immunized with the heterologous prime-boost regimens not only elicited stronger humoral responses but also induced stronger cellular responses than those receiving the homologous regimens.

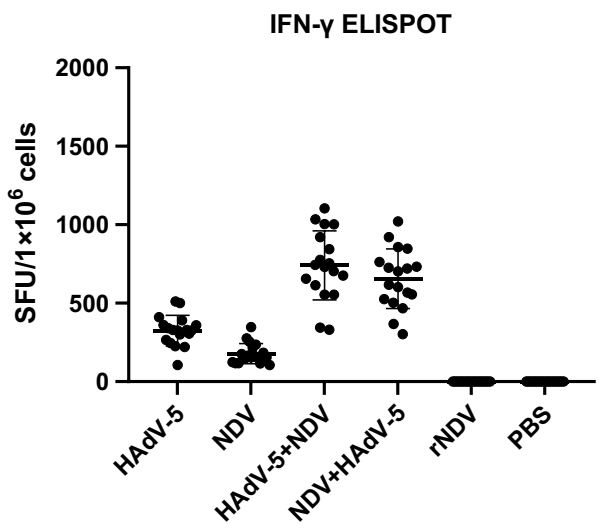

Fig. 6 EBOV-GP-specific IFN- $\gamma$ responses. Mice were sacrificed at 49 days post-inoculation. The splenocytes were isolated and assayed by ELISPOT for an EBOV-GP-specific IFN- $\gamma$ response

\section{Discussion}

In the present study, we successfully generated an NDV LaSota strain-based recombinant virus, rLS/EB-GP, expressing the GP protein of the EBOV variant Makona, as a vaccine candidate, using reverse genetics techniques. The immune responses of mice to this rLS/EB-GP recombinant vaccine and an HAdV-5-based EBOV experimental vaccine were evaluated by prime-boost regimens. The ELISA and ELISPOT results indicated that heterologous prime-boost regimens elicit more-robust immune responses than homologous regimens. The HAdV-5 prime 
and rLS/EB-GP boost immunization strategy was found to be the most effective approach for stimulating EBOVspecific immunities among the different regimens.

Human adenovirus has emerged as an ideal vaccine vector because of its relative safety, high transgene expression levels, and its capacity to elicit robust humoral and cellular immune responses to the encoded antigens. [30]. However, serosurveillance has shown that PEI to HadV-2 and HAdV-5 existed in up to $50 \%$ of the American population and up to 95\% in people from other countries. This high seroprevalence limits the efficacy of adenovirus (Ad)-vectored vaccines [4]. To circumvent the problem of PEI interference and improve the effectiveness of Ad-based vaccines, researchers have developed non-human primate (e.g., chimpanzee and gorilla) adenovirus vectors [6], explored different immunization routes [22], and, notably, investigated prime-boost regimens with heterologous vectors [18]. Regarding the last of these, studies have demonstrated that heterologous primeboost regimens employing a DNA prime and an HAdV-5 or ??modified vaccinia virus Ankara?? (MVA) boost could effectively induce humoral and cellular immune responses, and more importantly, improve vaccine efficacy, regardless of pre-existing Ad or MVA immunity [14, 29]. Three EBOV vaccine candidates adopting the heterologous prime-boost strategy have undergone different phases of clinical trials [20]. Results from these investigations indicated that both heterologous vector vaccine regimens of ChAd3-EBOV and Ad26.ZEBOV vaccines boosted with MVA elicited stronger B-cell and T-cell immune responses to EBOV than those induced by single-dose prime vaccination alone $[2,10]$.

EBOV transmission occurs exclusively through contact with mucosal surfaces. It might therefore be advantageous to use a vaccine vector with a natural tropism for the respiratory tract, which could induce the first line of defense at mucosal points of entry and robust systemic immune responses in the respiratory tract. Numerous researches have shown that NDV, an avian paramyxovirus, is an attractive vaccine vector for human pathogens, inducing humoral and cellular immune responses at both the mucosal and systemic levels in murine and non-human primate models [15]. Therefore, in this study, we adopted NDV as a vaccine vector to express the EBOV GP protein as a heterologous boost vaccine with the hope of avoiding the PEI interference associated with Ad-based primed vaccines. The results presented in this report are in agreement with the findings from the EBOV vaccine trials using the heterologous prime-boost immunization strategy [20], confirming that heterologous prime-boost regimens can elicit more-robust humoral and cellular responses to EBOV than homologous regimens [10, 26].

We observed with the homologous regimens that although two doses of rLS/EB-GP or HAdV-5 induced detectable EBOV-GP-specific antibody and T-cell responses in mice, the immune responses elicited by HAdV-5 were twofold higher than those induced by NDV. This difference might be due to differences in the amount of antigen expressed/ presented by these two vectors or the different immunization routes used to stimulate the immune system. Furthermore, the DNA vector HAdV-5 and the RNA vector NDV undoubtedly stimulate innate pathways differently. Therefore, we adopted the non-uniform but normal dosage of HAdV-5 (1 $\times 10^{7}$ IFU) and NDV $\left(1 \times 10^{6} \mathrm{PFU}\right)$ in this study. Further studies may be needed to determine whether a higher dose of NDV could enhance vaccine effectiveness.

In contrast to the homologous regimens, the heterologous prime-boost with HAdV-5 and rLS/EB-GP induced stronger immune responses than the homologous regimens. The most robust EBOV-GP-specific antibody and T-cell responses were detected by ELISA and ELISPOT in the HAdV-5 prime and NDV boost group, while the reversed NDV prime-HAdV-5 boost ranked second. The reason for this difference is still unclear. A possible explanation may be differences in the way stimulating innate immunity is stimulated by an RNA virus (NDV) and a DNA virus (Ad), which would affect the direction and strength of the subsequent adaptive immune response. This hypothesis needs to be tested in future studies by analyzing markers of immune responses.

Funding This research was supported by the Cultivation Fund of the Beijing Center for Disease Prevention and Control, Beijing Research Center for Preventive Medicine (grant no. 2017-BJYJ-13).

\section{Declarations}

Conflict of interest No competing financial interests exist.

Ethical approval Animal usage protocols used in this study were approved by the Beijing Center for Disease Prevention and Control (CDC) Institutional Animal Care and Use Committee (IACUC). At the termination of the experiments, all animals were humanely euthanized in accordance with Beijing-CDC-IACUC-approved animal usage protocols.

\section{References}

1. Alexander DJ (2000) Newcastle disease and other avian paramyxoviruses. Rev Sci Tech 19:443-462

2. Anywaine Z, Whitworth H, Kaleebu P, Praygod G, Shukarev G, Manno D, Kapiga S, Grosskurth H, Kalluvya S, Bockstal V, Anumendem D, Luhn K, Robinson C, Douoguih M, Watson-Jones D (2019) Safety and immunogenicity of a 2-dose heterologous vaccination regimen with Ad26.ZEBOV and MVA-BN-Filo Ebola vaccines: 12 -month data from a phase 1 randomized clinical trial in Uganda and Tanzania. J Infect Dis 220:46-56

3. Barouch DH, Pau MG, Custers JH, Koudstaal W, Kostense S, Havenga MJ, Truitt DM, Sumida SM, Kishko MG, Arthur JC, 
Korioth-Schmitz B, Newberg MH, Gorgone DA, Lifton MA, Panicali DL, Nabel GJ, Letvin NL, Goudsmit J (2004) Immunogenicity of recombinant adenovirus serotype 35 vaccine in the presence of pre-existing anti-Ad5 immunity. J Immunol 172:6290-6297

4. Barouch DH (2010) Novel adenovirus vector-based vaccines for HIV-1. Curr Opin HIV AIDS 5:386-390

5. Bausch DG, Towner JS, Dowell SF, Kaducu F, Lukwiya M, Sanchez A, Nichol ST, Ksiazek TG, Rollin PE (2007) Assessment of the risk of Ebola virus transmission from bodily fluids and fomites. J Infect Dis 196(Suppl 2):S142-147

6. Capone S, D'Alise AM, Ammendola V, Colloca S, Cortese R, Nicosia A, Folgori A (2013) Development of chimpanzee adenoviruses as vaccine vectors: challenges and successes emerging from clinical trials. Expert Rev Vaccines 12:379-393

7. Coltart CE, Lindsey B, Ghinai I, Johnson AM, Heymann DL (2017) The Ebola outbreak, 2013-2016: old lessons for new epidemics. Philos Trans R Soc Lond B Biol Sci 372:12-35

8. DiNapoli JM, Kotelkin A, Yang L, Elankumaran S, Murphy BR, Samal SK, Collins PL, Bukreyev A (2007) Newcastle disease virus, a host range-restricted virus, as a vaccine vector for intranasal immunization against emerging pathogens. Proc Natl Acad Sci USA 104:9788-9793

9. Estevez C, King D, Seal B, Yu Q (2007) Evaluation of Newcastle disease virus chimeras expressing the Hemagglutinin-Neuraminidase protein of velogenic strains in the context of a mesogenic recombinant virus backbone. Virus Res 129:182-190

10. Ewer K, Rampling T, Venkatraman N, Bowyer G, Wright D, Lambe T, Imoukhuede EB, Payne R, Fehling SK, Strecker T, Biedenkopf N, Krahling V, Tully CM, Edwards NJ, Bentley EM, Samuel D, Labbe G, Jin J, Gibani M, Minhinnick A, Wilkie M, Poulton I, Lella N, Roberts R, Hartnell F, Bliss C, SierraDavidson K, Powlson J, Berrie E, Tedder R, Roman F, De Ryck I, Nicosia A, Sullivan NJ, Stanley DA, Mbaya OT, Ledgerwood JE, Schwartz RM, Siani L, Colloca S, Folgori A, Di Marco S, Cortese R, Wright E, Becker S, Graham BS, Koup RA, Levine MM, Volkmann A, Chaplin P, Pollard AJ, Draper SJ, Ballou WR, Lawrie A, Gilbert SC, Hill AV (2016) A Monovalent Chimpanzee Adenovirus Ebola Vaccine Boosted with MVA. N Engl J Med 374:1635-1646

11. Freeman AI, Zakay-Rones Z, Gomori JM, Linetsky E, Rasooly L, Greenbaum E, Rozenman-Yair S, Panet A, Libson E, Irving CS, Galun E, Siegal T (2006) Phase I/II trial of intravenous NDV-HUJ oncolytic virus in recurrent glioblastoma multiforme. Mol Ther 13:221-228

12. Hu H, Roth JP, Estevez CN, Zsak L, Liu B, Yu Q (2011) Generation and evaluation of a recombinant Newcastle disease virus expressing the glycoprotein $(\mathrm{G})$ of avian metapneumovirus subgroup $\mathrm{C}$ as a bivalent vaccine in turkeys. Vaccine 29:8624-8633

13. Kardani K, Bolhassani A, Shahbazi S (2016) Prime-boost vaccine strategy against viral infections: Mechanisms and benefits. Vaccine 34:413-423

14. Kibuuka H, Kimutai R, Maboko L, Sawe F, Schunk MS, Kroidl A, Shaffer D, Eller LA, Kibaya R, Eller MA, Schindler KB, Schuetz A, Millard M, Kroll J, Dally L, Hoelscher M, Bailer R, Cox JH, Marovich M, Birx DL, Graham BS, Michael NL, de Souza MS, Robb ML (2010) A phase 1/2 study of a multiclade HIV-1 DNA plasmid prime and recombinant adenovirus serotype 5 boost vaccine in HIV-Uninfected East Africans (RV 172). J Infect Dis 201:600-607

15. Kim SH, Samal SK (2016) Newcastle disease virus as a vaccine vector for development of human and veterinary vaccines. Viruses 8:183-207

16. Kolakofsky D, Roux L, Garcin D, Ruigrok RWH (2005) Paramyxovirus mRNA editing, the "rule of six" and error catastrophe: a hypothesis. J Gen Virol 86:1869-1877
17. Li JX, Hou LH, Meng FY, Wu SP, Hu YM, Liang Q, Chu K, Zhang Z, Xu JJ, Tang R, Wang WJ, Liu P, Hu JL, Luo L, Jiang $\mathrm{R}$, Zhu FC, Chen W (2017) Immunity duration of a recombinant adenovirus type- 5 vector-based Ebola vaccine and a homologous prime-boost immunisation in healthy adults in China: final report of a randomised, double-blind, placebo-controlled, phase 1 trial. Lancet Glob Health 5:e324-e334

18. Logunov DY, Dolzhikova IV, Zubkova OV, Tukhvatulin AI, Shcheblyakov DV, Dzharullaeva AS, Grousova DM, Erokhova AS, Kovyrshina AV, Botikov AG, Izhaeva FM, Popova O, Ozharovskaya TA, Esmagambetov IB, Favorskaya IA, Zrelkin DI, Voronina DV, Shcherbinin DN, Semikhin AS, Simakova YV, Tokarskaya EA, Lubenets NL, Egorova DA, Shmarov MM, Nikitenko NA, Morozova LF, Smolyarchuk EA, Kryukov EV, Babira VF, Borisevich SV, Naroditsky BS, Gintsburg AL (2020) Safety and immunogenicity of an rAd26 and rAd5 vector-based heterologous prime-boost COVID-19 vaccine in two formulations: two open, non-randomised phase 1/2 studies from Russia. Lancet 396:887-897

19. Martin DA, Muth DA, Brown T, Johnson AJ, Karabatsos N, Roehrig JT (2000) Standardization of immunoglobulin M capture enzyme-linked immunosorbent assays for routine diagnosis of arboviral infections. J Clin Microbiol 38:1823-1826

20. Matz KM, Marzi A, Feldmann H (2019) Ebola vaccine trials: progress in vaccine safety and immunogenicity. Expert Rev Vaccines 18:1229-1242

21. Nyombayire J, Anzala O, Gazzard B, Karita E, Bergin P, Hayes P, Kopycinski J, Omosa-Manyonyi G, Jackson A, Bizimana J, Farah B, Sayeed E, Parks CL, Inoue M, Hironaka T, Hara H, Shu T, Matano T, Dally L, Barin B, Park H, Gilmour J, Lombardo A, Excler JL, Fast P, Laufer DS, Cox JH, Team SS (2017) First-inhuman evaluation of the safety and immunogenicity of an intranasally administered replication-competent sendai virus-vectored HIV type 1 gag vaccine: induction of potent T-cell or antibody responses in prime-boost regimens. J Infect Dis 215:95-104

22. Richardson JS, Pillet S, Bello AJ, Kobinger GP (2013) Airway delivery of an adenovirus-based Ebola virus vaccine bypasses existing immunity to homologous adenovirus in nonhuman primates. J Virol 87:3668-3677

23. Samal SK (2011) Newcastle disease and related avian paramyxoviruses. Caister Academic Press, Norfolk

24. Stanley DA, Honko AN, Asiedu C, Trefry JC, Lau-Kilby AW, Johnson JC, Hensley L, Ammendola V, Abbate A, Grazioli F, Foulds KE, Cheng C, Wang L, Donaldson MM, Colloca S, Folgori A, Roederer M, Nabel GJ, Mascola J, Nicosia A, Cortese R, Koup RA, Sullivan NJ (2014) Chimpanzee adenovirus vaccine generates acute and durable protective immunity against ebolavirus challenge. Nat Med 20:1126-1129

25. Sullivan NJ, Sanchez A, Rollin PE, Yang ZY, Nabel GJ (2000) Development of a preventive vaccine for Ebola virus infection in primates. Nature 408:605-609

26. Tapia MD, Sow SO, Lyke KE, Haidara FC, Diallo F, Doumbia M, Traore A, Coulibaly F, Kodio M, Onwuchekwa U, Sztein MB, Wahid R, Campbell JD, Kieny MP, Moorthy V, Imoukhuede EB, Rampling T, Roman F, De Ryck I, Bellamy AR, Dally L, Mbaya OT, Ploquin A, Zhou Y, Stanley DA, Bailer R, Koup RA, Roederer M, Ledgerwood J, Hill AVS, Ballou WR, Sullivan N, Graham B, Levine MM (2016) Use of ChAd3-EBO-Z Ebola virus vaccine in Malian and US adults, and boosting of Malian adults with MVA-BN-Filo: a phase 1, single-blind, randomised trial, a phase $1 \mathrm{~b}$, open-label and double-blind, dose-escalation trial, and a nested, randomised, double-blind, placebo-controlled trial. Lancet Infect Dis 16:31-42

27. Thacker EE, Timares L, Matthews QL (2009) Strategies to overcome host immunity to adenovirus vectors in vaccine development. Expert Rev Vaccines 8:761-777 
28. Wu S, Kroeker A, Wong G, He S, Hou L, Audet J, Wei H, Zhang Z, Fernando L, Soule G, Tran K, Bi S, Zhu T, Yu X, Chen W, Qiu X (2016) An Adenovirus vaccine expressing Ebola virus variant Makona glycoprotein is efficacious in guinea pigs and nonhuman primates. J Infect Dis 214:S326-S332

29. Yang ZY, Wyatt LS, Kong WP, Moodie Z, Moss B, Nabel GJ (2003) Overcoming immunity to a viral vaccine by DNA priming before vector boosting. J Virol 77:799-803

30. Zhang C, Zhou D (2016) Adenoviral vector-based strategies against infectious disease and cancer. Hum Vaccin Immunother 12:2064-2074

31. Zhao W, Spatz S, Zhang Z, Wen G, Garcia M, Zsak L, Yu Q (2014) Newcastle disease virus (NDV) recombinants expressing infectious laryngotracheitis virus (ILTV) glycoproteins $\mathrm{gB}$ and gD protect chickens against ILTV and NDV challenges. J Virol 88:8397-8406
32. Zhao W, Zhang ZY, Zsak L, Yu QZ (2015) P and M gene junction is the optimal insertion site in Newcastle disease virus vaccine vector for foreign gene expression. J Gen Virol 96:40-45

33. Zhu FC, Hou LH, Li JX, Wu SP, Liu P, Zhang GR, Hu YM, Meng FY, Xu JJ, Tang R, Zhang JL, Wang WJ, Duan L, Chu K, Liang Q, Hu JL, Luo L, Zhu T, Wang JZ, Chen W (2015) Safety and immunogenicity of a novel recombinant adenovirus type-5 vectorbased Ebola vaccine in healthy adults in China: preliminary report of a randomised, double-blind, placebo-controlled, phase 1 trial. Lancet 385:2272-2279

Publisher's Note Springer Nature remains neutral with regard to jurisdictional claims in published maps and institutional affiliations. 